\title{
Two cases of rickets presenting with poor growth, hypotonia, and respiratory problems
}

\author{
E. Wouters ${ }^{1,2}$, M. Wojciechowski ${ }^{1}$, E. de Vries $^{2}$ \\ ${ }^{1}$ Antwerp University Hospital, University of Antwerp, Belgium, ${ }^{2}$ Jeroen Bosch Hospital, The Netherlands
}

Rickets is a rare disease in developed countries. In children, it is a disease which affects growing bone. Depending on the severity, it can present with a wide variety of symptoms. Because it is such a rare disease in developed countries, symptoms suggesting rickets are often not easily recognized. This can cause a delay in diagnosing and treating rickets. Often unnecessary and sometimes invasive investigations are performed. First leading clues to rickets on physical examination are poor growth, especially length, thickening of wrists, bow legs, and craniotabes. At further examination, special attention should be paid to osteopenia and cupping and fraying at the metaphyses on X-rays. Laboratory results suggestive for rickets are elevated alkaline phosphatase and disturbances in calcium and phosphate homeostasis. In this report, we present two cases presenting with poor growth, severe pain, and respiratory problems secondary to calcipenic rickets.

Keywords: Rickets, Failure to thrive, Vitamin D, Osteopenia, Poor growth

\section{Introduction}

In everyday practice, a paediatrician is often challenged with a child suffering poor growth. This is a difficult symptom; the cause is not always clear. In more severe cases, this is called stunting which is defined by WHO as height for age-2 SD of the WHO child growth standards median. Growth retardation may be associated with 'failure to thrive', which is defined as weight that falls below the fifth percentile or deceleration that crosses two major percentiles on a growth chart. In mild cases of failure to thrive, linear growth is preserved. In the most severe cases, however, linear growth and even head circumference may also be affected. The cause of poor growth can be unexpected, as we show in the following cases.

\section{Case 1}

A 1.5-year-old Somalian boy was presented in the outpatient clinic because of poor growth and persistent cough. His medical history was, besides recurrent episodes of fever, unremarkable. He was exclusively breastfed; he did not get any supplements. He was born in the Netherlands, and had never been abroad. There was no known contact with infections. Physical examination showed a mildly hypotonic, dystrophic child with respiratory distress and some hoarse crackles over the lungs. His weight was at the

Correspondence to: E. Wouters, Department of paediatrics, University Hospital Antwerp, Wilrijkstraat 10, 2650 Antwerp, Belgium. Email: Edwardwouters@hotmail.com second centile, his length below the third centile, and his head circumference at the fiftieth centile. The boy was admitted for further analysis and treatment. Investigations, with a focus on lower respiratory tract infection, potential malabsorption, immunodeficiency, and intake problems, were undertaken. Because of the persistent cough, pneumonia was suspected. X-ray showed a chest infiltration with atelectasis. Bacterial culture of broncho-alveolar lavage fluid grew Streptococcus pneumoniae for which oral amoxicillin was started. The tuberculin skin test was negative. Laboratory results revealed low serum calcium (2.14 mmol/1; normal: 2.14-2.64 mmol/l) and phosphate (0.68 mmol/l; normal: $1.2-2.1 \mathrm{mmol} / \mathrm{l})$, and high alkaline phosphatase (773 U/1; 60-420 U/1). These results combined with his short stature suggested rickets. On repeated physical examination thickened wrists were found. X-ray of the long bones revealed cupping and fraying of the metaphyses. $25-\mathrm{OH}$ vitamin D was $13.0 \mathrm{pmol} / \mathrm{l}(50-120 \mathrm{pmol} / \mathrm{l})$ and PTH was 139.0 pmol (1.3-6.8 pmol/l), suggesting rickets caused by vitamin $\mathrm{D}$ deficiency, probably due to lack of vitamin D prophylaxis. The other laboratory investigations, including celiac serology, immunophenotyping, mycoplasma, and HIV serology, were unremarkable. No parasites were found in the faeces.

Treatment was started with high-dose vitamin D in combination with phosphate and calcium. His clinical condition quickly improved. Laboratory findings normalized and X-ray of the long bones showed improved mineralisation. After several months, cal- 
cium and phosphate suppletion were stopped, and only advised doses of vitamin $\mathrm{D}$ were given.

\section{Case 2}

Monochorionic diamniotic twins were referred at the age of 10 months with respiratory distress, feeding problems, and severe hypotonia. Apart from premature birth at 30 weeks of gestational age with an uncomplicated course, there were no major problems in the history. They were born in Belgium and had never been abroad. Vitamin D prophylaxis was given in a proper dose. The parents were from West-African origin; the family history was unremarkable. The parents were not consanguineous. Physical examination showed very painful, severely hypotonic twins with bell-shaped chests, pronounced muscle weakness, and severe respiratory distress. Craniotabes, thickened joints, and bowing of the legs were found. Their weight was at the third centile; their length and head circumferences were far below the third centile. Rickets was immediately suspected because of the craniotabes and thickened joints. X-ray showed a chest infiltrate with atelectasis, and costochondral swelling with a typical rosary pattern. X-ray of the long bones showed severe osteopenia and cupping and fraying of the metaphyses. $25-\mathrm{OH}$ vitamin $\mathrm{D}$ was $45 \mathrm{ng} / \mathrm{ml} \quad(30-100 \mathrm{ng} / \mathrm{ml})$, calcium $9.5 \mathrm{mg} / \mathrm{dl} \quad(8.0-$ $11.4 \mathrm{mg} / \mathrm{dl})$, phosphate $2.1 \mathrm{mg} / \mathrm{dl}(1.2-2.1 \mathrm{mg} / \mathrm{l})$, and alkaline phosphatase $4107 \mathrm{U} / \mathrm{l}(60-420 \mathrm{U} / \mathrm{l}) \cdot 1.25-\mathrm{OH}_{2}$ Vitamin D was $202 \mathrm{pg} / \mathrm{ml}(20-75 \mathrm{pg} / \mathrm{ml})$. These results suggested a vitamin D resistant form of rickets.

Treatment with high-dose alphacalcidol in combination with phosphate and calcium was started. For the pneumonia, supplementary oxygen and intravenous amoxicillin were given. The pain disappeared and the respiratory problems slowly subsided with improving of the hypotonia and muscle weakness. Within a few months, the osteopenia on X-ray diminished and laboratory findings normalized. DNA sequence analysis for vitamin-D-receptor-gene was negative. Further analysis of the underlying defect was not performed so far.

\section{Discussion}

Poor growth can be a diagnostic challenge for a paediatrician. Because the causes are so many, detailed history and physical examination including complete biometry are very important for achieving the right diagnosis. Often, simple investigations can provide important information. Calcipenic rickets, as in our two cases, is a very rare cause for poor growth. Because rickets in itself is such a rare disease nowadays in developing countries, signs and symptoms are not always recognized or are misinterpreted.

Rickets is a disease of growing bone and therefore unique to children and adolescents. It refers to a failure of mineralization of growing bone and cartilage. Although it is a serious condition, rickets caused by vitamin $\mathrm{D}$ deficiency can easily be treated. So, recognition is important.

There are four different mechanisms which can cause calcipenic rickets. Rickets is most often due to vitamin D deficiency caused by low dietary intake and/or low sun exposure. Two-thirds of the daily requirement of vitamin $\mathrm{D}$ is obtained from exposure to sunlight. The other one-third comes from dietary vitamin $\mathrm{D}$, originating only from animal source foods. Rarer causes are hydroxylation problems of vitamin D precursors to $25-\mathrm{OH}$ vitamin $\mathrm{D}$ in the liver (storage form of vitamin $\mathrm{D}$ ), problems of hydroxylation of $25-\mathrm{OH}$ to $1,25-\mathrm{OH}_{2}$ vitamin $\mathrm{D}$ in the kidney (active metabolite of vitamin D) and finally insensibility of the target organ to vitamin D (Fig. 1). This last one is called hereditary vitamin D-resistant rickets or vitamin-D-dependant rickets type II. It is a rare form of calcipenic rickets caused by a gene mutation that encodes the vitamin $\mathrm{D}$ receptor. It is characterized by end-organ resistance to vitamin D resulting in normal 25-OH vitamin D levels and very high $1,25-\mathrm{OH}_{2}$ vitamin D. So far, there are about 100 cases described with this form of rickets.

Nowadays, with the advice for vitamin D prophylaxis, rickets due to vitamin $\mathrm{D}$ deficiency is a rare disease in developed countries. On the other hand, the frequency of vitamin D deficiency has been increasing the last years. Probable causes for this increase are the recommendation to apply sunscreen, a tendency for children to spend more time inside playing computer games or watching television and atmospheric pollution limiting ultraviolet radiation reaching the skin. ${ }^{1}$ Furthermore, in the growing multicultural society, we are increasingly confronted with different cultures. Certain cultural groups are at risk for vitamin D deficiency: dark-skinned children need more sunlight exposure; ${ }^{2}$ belief can make parents not give their child supplemental vitamin D; prolonged and exclusive breastfeeding without supplementation, ${ }^{3}$ clothing, and different diet (lack of animal source foods) can contribute to low vitamin D levels. These causes together ensure that rickets still can be seen in developed countries.

Depending on the severity, rickets can present with a wide variety of symptoms. The child may be asymptomatic or present with varying degrees of pain and irritability, motor development delay, poor growth, and increased susceptibility to (respiratory) infections. Clinical findings of rickets in infants and younger children include craniotabes (areas of thinning and softening of bones of the skull), delayed closure of the fontanel, frontal bossing, wrist widening, and bow legs. In older children, the most visible symptoms are symptoms caused by weight bearing deformities such as bowing of the legs, prominence of costochondral 


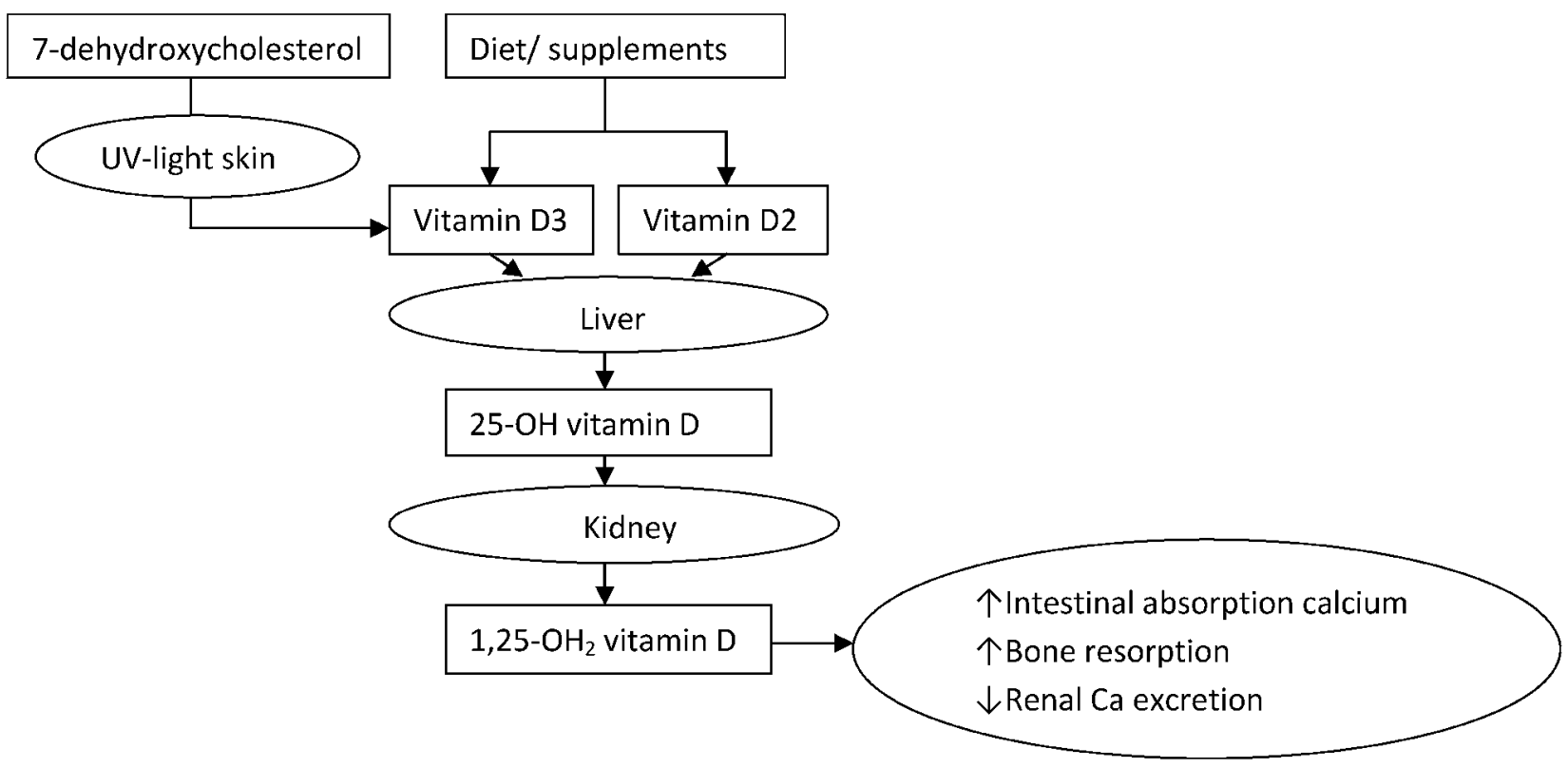

Figure 1 The vitamin D precursors (vitamin D2 and D3) are obtained from dietary intake and by exposure to ultraviolet radiation where 7-dehydroxycholesterol is synthesized to vitamin D3. The vitamin $D$ precursors then are hydroxylated in the liver to the storage form of vitamin $\mathrm{D}, 25-\mathrm{OH}-\mathrm{Vitamin} \mathrm{D}$. The inactive 25-OH-vitamin $\mathrm{D}$ is hydroxylated in the kidney to the active metabolite of vitamin $\mathrm{D}, 1,25-\mathrm{OH}_{2}$ vitamin $\mathrm{D}$. 1,25- $\mathrm{OH}_{2}$ vitamin $\mathrm{D}$ causes intestinal calcium absorption, bone resorption and renal Ca excretion.

junctions and knock-knees: these last two are caused by laying down of uncalcified osteoid at the metaphyses leading to spreading of those areas and producing knobby deformity. Besides the skeletal aspects, vitamin $\mathrm{D}$ deficiency causes extra-skeletal signs and symptoms, including muscle weakness and generalized hypotonia, delayed motor development, convulsions, sweating, and disturbed immunity.

In the described cases, the poor increase of length and weight was severe. Unlike in children with failure to thrive, in children with rickets, the first growth deflection will be in length instead of in weight due to failure in bone mineralization. In both cases, the children initially presented with hypotonia, feeding problems and respiratory problems. In case 1, first investigations were aimed for causes of failure to thrive, chronic respiratory infections, and immune deficiency disorders. A first leading clue to rickets, besides the small length, could have been found on physical examination. Unfortunately, the thickened joints were initially not recognized. In case 2 , the children were seen several times with respiratory and feeding problems by a paediatrician, before they were referred to the paediatric neurologist for evaluation of hypotonia and muscle weakness. The difference in weight and length deflection was not noticed. In these children, after referral craniotabes was found on physical examination and rickets was immediately suspected. Further investigation was focused on rickets.

Rickets is a clinical diagnosis which can be confirmed by radiological examination. Laboratory testing allows further differentiating the cause of rickets. Alkaline phosphatase is usually highly elevated in rickets. In case 1, probably because rickets was not suspected when the first chest X-ray was made, the costochondral abnormalities and osteopenia were not detected. Rickets was suspected after combining the abnormal laboratory findings (low to normal calcium, low phosphate, and highly elevated alkaline phosphatase) together with the short stature. Rickets was confirmed by X-ray of the long bones. Reviewing the first chest-X-ray, the osteopenia and rib abnormalities already could be seen. In case 2, imaging confirmed the clinical suspicion for rickets. In case 1 , rickets was caused by $25-\mathrm{OH}$ vitamin $\mathrm{D}$ deficiency due to dietary insufficiency. The diagnosis was confirmed after 2 weeks. In case 2, suspicions for vitamin D resistant rickets arose because of the extremely elevated alkaline phosphatase with normal calcium and phosphate and normal vitamin D.

In these cases, the presenting respiratory symptoms were presumed to be caused by hypotonia due to vitamin D deficiency. Recent literature suggests a relation between vitamin $\mathrm{D}$ and the immune system. ${ }^{4}$ When treatment was started, the hypotonia and pulmonary problems resolved in all three children. The child in case 1 recovered completely gaining normal height and weight. The twins improved tremendously with disappearance of pain and regaining of normal strength. Although they showed catchup growth, normal length has not been gained so far. In the latter case, the children, as a result of the cause, were more severely affected and needed much higher doses of vitamin $\mathrm{D}$, calcium, and phosphate. 


\section{Conclusion}

Rickets is a rare cause for growth retardation. Despite the advice for vitamin D prophylaxis, it still can be seen in developed countries. In children with poor growth, unexplained pain, hypotonia, or respiratory problems, rickets should be considered. Biometry together with good physical examination can give a first clue. On physical examination, special attention should be given to specific symptoms such as craniotabes, thickening of wrists, knuckle knees, and bow legs. When additional investigations are performed, it is important to look for signs of osteopenia on X-rays and for laboratory findings suggesting rickets. Treatment with vitamin $\mathrm{D}$, calcium, and phosphate can be started early and, depending on the cause, complete recovery can be gained.

\section{Disclaimer Statements}

Contributors EW had primary responsibility for assessment of cases and prepared the manuscript.
MW, and EDV had responsibility for assessment of the cases and revised the manuscript.

Funding None.

Conflicts of interest The authors declare no conflicts of interest.

\section{Ethics approval None.}

\section{References}

1 Agarwal KS, Mughal MZ, Upadhyay P, Berry JL, Mawer EB, Puliyel JM. The impact of atmospheric pollution on vitamin D status of infants and toddlers in Delhi, India. Arch Dis Child. 2002;87:111-3.

2 Clemens TL, Adams JS, Henderson SL, Holick MF. Increased skin pigmentation reduces the capacity of skin to synthesize vitamin D3. Lancet. 1982;1:74-6.

3 Mughal MZ, Salama H, Greenaway T, Laing I, Mawer EB. Florid rickets associated with prolonged breast feeding without vitamin D supplementation. BMJ. 1999; 318:39-40

4 Shaw NJ, Mughal MZ. Vitamin D and child health: part 2 (extraskeletal and other aspects). Arch Dis Child. 2013;98: 363-7. 